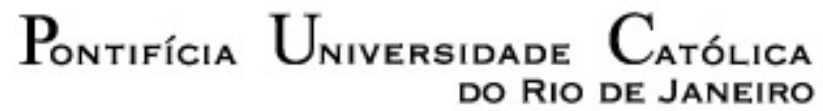

DO RIO DE JANEIRO

Tarcísio de Freitas Cardoso

Visão geral sobre espectros de resposta sísmica para sistemas secundários

Dissertação de Mestrado

Dissertação apresentada como requisito parcial para obtenção do título de Mestre pelo Programa de PósGraduação em Engenharia Civil da PUC-Rio.

Orientador: João Luis Pascal Roehl Co-orientadora: Andréia Abreu Diniz de Almeida

Rio de Janeiro, setembro de 2008 


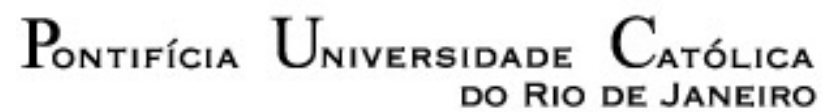

DO RIO DE JANEIRO

Tarcísio de Freitas Cardoso

\section{Visão geral sobre espectros de resposta sísmica para sistemas secundários}

Dissertação apresentada como requisito parcial para obtenção do título de Mestre pelo Programa de PósGraduação em Engenharia Civil da PUC-Rio. Aprovada pela Comissão Examinadora abaixo assinada.

João Luis Pascal Roehl

Orientador

Departamento de Engenharia Civil - PUC-Rio

Andréia Abreu Diniz de Almeida

Co-orientadora

Departamento de Engenharia Civil - PUC-Rio

Tereza Denyse Pereira de Araújo Universidade Federal do Ceara - UFC

Rodolfo Luiz Martins Suanno Universidade do Estado do Rio de Janeiro - UERJ

Raul Rosas e Silva

Departamento de Engenharia Civil - PUC-Rio

José Eugenio Leal

Coordenador Setorial do Centro Técnico Científico - PUC-Rio

Rio de Janeiro, 08 de setembro de 2008 
Todos os direitos reservados. É proibida a reprodução total ou parcial do trabalho sem autorização da universidade, do autor e do orientador.

\section{Tarcísio de Freitas Cardoso}

Graduou-se em Engenharia Civil, pela Universidade Federal do Rio de Janeiro em dezembro de 1980. Cursou pósgraduação em Mecânica dos Solos, na COPPE/UFRJ, pelo projeto Urânio em 1981. Ingressou na NUCLEN em março de 1982, atuando nas áreas de Análise Sísmica e de Estruturas metálicas. Atualmente trabalha na ELETRONUCLEAR, na Gerência de Análise de Tensões, atuando na área de dinâmica das estruturas.

Ficha Catalográfica

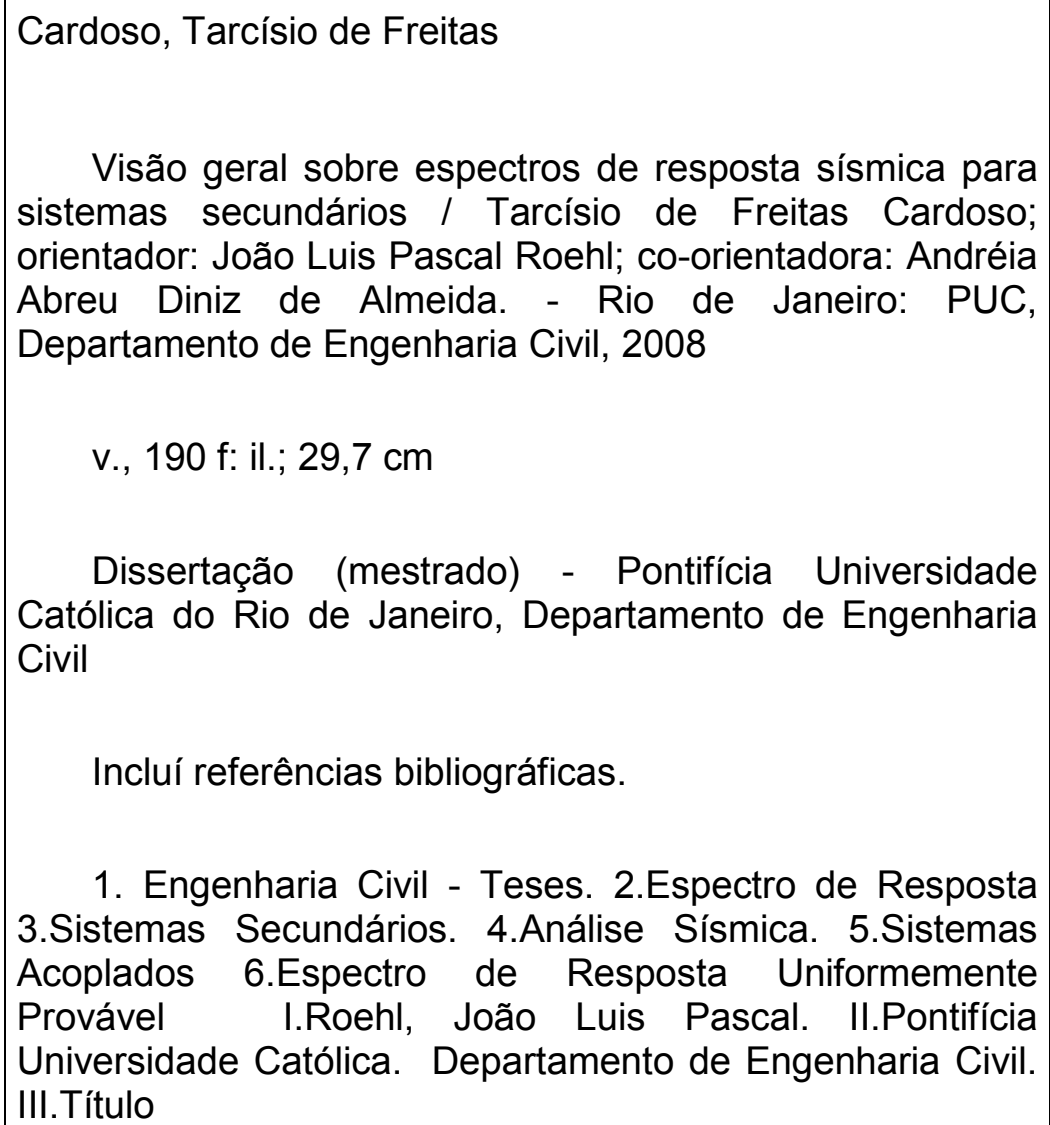

Visão geral sobre espectros de resposta sísmica para sistemas secundários / Tarcísio de Freitas Cardoso; orientador: João Luis Pascal Roehl; co-orientadora: Andréia Abreu Diniz de Almeida. - Rio de Janeiro: PUC, Departamento de Engenharia Civil, 2008

v., 190 f: il.; $29,7 \mathrm{~cm}$

Dissertação (mestrado) - Pontifícia Universidade Católica do Rio de Janeiro, Departamento de Engenharia Civil

Incluí referências bibliográficas.

1. Engenharia Civil - Teses. 2.Espectro de Resposta 3.Sistemas Secundários. 4.Análise Sísmica. 5.Sistemas Acoplados 6.Espectro de Resposta Uniformemente Provável I.Roehl, João Luis Pascal. II.Pontifícia Universidade Católica. Departamento de Engenharia Civil. III.Título 


\section{"PARA SER GRANDE, sê inteiro: nada \\ TEU exagera ou exclui.}

Sê todo em cada coisa. Põe quanto és

No mínimo que fazes.

Assim em cada lago a lua toda

Brilha, porque alta vive."

(Fernando Pessoa, como Ricardo Reis 14/2/1933)

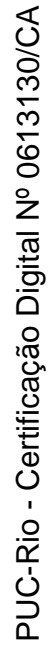

A meu pai, Edyo Cardoso.

$\mathrm{O}$ exemplo permanece. 


\section{Agradecimentos}

À Irene, pela presença, cumplicidade e partilha de vida, que nos fazem tão felizes.

À minha mãe, Taïs, que com seu carinho, compreensão e firmeza continua me educando para a vida.

Ao prof. Roehl, pela dedicação, compreensão, transmissão de conhecimentos e principalmente pelos sábios ensinamentos de vida, mais profundos e importantes.

À ELETRONUCLEAR e à PUC-Rio, pela confiança e suporte.

Aos professores e funcionários do DEC, compreensivos, prestativos e atenciosos.

Aos colegas Marcelo, Waldo, Marcos, Regina, Andréia, Denyse e Jair, que me antecederam na pesquisa e cujo trabalho apenas complemento. Aos colegas da ETN, principalmente os mais próximos e os da GAN.T, pelo apoio e incentivo. Aos colegas da pós-graduação, pelo afeto e carinho, que fizeram as nossas atividades transcorrerem com alegria e esperança, fazendo-me sentir jovem novamente.

À Paôla e à Andréia, amigas, que foram estímulo constante e a parceria que tornou possível a realização desse trabalho.

Ao amigo Prates, incentivo permanente, pelas discussões e sugestões, e à Maria Teresa, pela revisão cuidadosa do texto.

Aos amigos e aos irmãos, pelo encorajamento e apoio imprescindível para superar os momentos difíceis, causados pelas tribulações ocorridas nos últimos anos.

Ao Tarcísio e ao Tiago, por serem a confirmação concreta do valor do empenho e atitude na tentativa de um mundo melhor.

A Quem é a origem da VIDA, que é bela de se viver. 


\section{Resumo}

Cardoso, Tarcísio de Freitas. Visão geral sobre espectros de resposta sísmica para sistemas secundários. Rio de Janeiro, 2008. 223p. Dissertação de Mestrado - Departamento de Engenharia Civil, Pontifícia Universidade Católica do Rio de Janeiro.

A indústria de geração elétrica de fonte nuclear baseia-se em princípios de segurança e, nos critérios de projeto, considera-se a hipótese de terremoto. Os sistemas necessários à segurança são projetados para resistir e manter a operabilidade durante e após eventos sísmicos postulados. Propõe-se um roteiro para a produção de espectros de resposta sísmica para projeto de sistemas secundários, SS, incluindo a influência do acoplamento e em base probabilística. O roteiro utiliza a ferramenta SASSI, pode ser utilizado em situações gerais de cálculo e fornece um conjunto de programas para considerar modelos tridimensionais e suas respostas para uma excitação genérica em 3 direções ortogonais; representar os efeitos de acoplamento entre o SS e o sistema principal, SP; incluir a influência dos deslocamentos relativos entre os nós de apoio do sistema secundário no sistema principal; utilizar os fatores de transposição entre espectros elásticos e inelásticos; permitir a análise probabilística e a obtenção de Espectros de Resposta Uniformemente Prováveis, acoplados ou não; incluir interfaces para a utilização de seus resultados com outros programas de utilização geral, como o MS-EXCEL. O elevado grau de automatização permite a produção de espectros de resposta com refinamentos de modelagem, alcançando uma análise mais realista, sem a necessidade de esforços adicionais aos já requeridos pela metodologia convencional. A metodologia proposta enquadra-se no encaminhamento para o contexto atual de análise sísmica de instalações nucleares, com a utilização de espectros de resposta de projeto de ameaça uniforme, específico para o sítio da instalação, e o projeto sísmico de risco consistente.

\section{Palavras-chave}

Análise Sísmica; Espectro de Resposta; Sistemas Secundários; Sistemas Secundários Acoplados; Espectro de Resposta Uniformemente Provável 


\section{Abstract}

Cardoso, Tarcísio de Freitas. Overview on secondary system seismic response spectra. Rio de Janeiro, 2008. 223p. Dissertação de Mestrado Departamento de Engenharia Civil, Pontifícia Universidade Católica do Rio de Janeiro.

The electric power reactor industry is based on rigid safety principles. The design criteria include seismic scenario. All safety related systems are designed to resist and to keep the operability during and after a postulated earthquake. It is suggested a procedure for the generation of in-structure seismic response spectra for secondary system design. A probabilistic approach is used and coupling effects between primary and secondary systems are taken into account. The proposed script uses SASSI system and can be used in general situations. A set of computer programs is developed to consider three-dimensional models and their responses for a generic base excitation, acting in 3 orthogonal directions; represent the coupling effect between primary and secondary systems, include the influence, on the response spectra, of the secondary system supports relative displacements; include approximated factors for transposition of elastic into inelastic response spectra; produce Uniformly Probable Response Spectra, including or not coupling effects; consider interfaces with other general programs, as the MS-EXCEL, for pos-processing purpose. The degree of automation, allows the production of response spectra including modeling refinements, reaching a more realistic analysis, without additional efforts beyond those already required by the conventional methodology. The proposed methodology is in the way of a site specific uniform hazard design response spectra, and of a consistent-risk seismic design.

\section{Keywords}

seismic analysis; response spectrum; secondary system; coupled secondary system; uniformly probable response spectra 


\section{Sumário}

1 Introdução 25

1.1. Aspectos Gerais 25

1.2. Pesquisas anteriores no DEC 28

1.3. Objetivos 30

1.4. Organização do Texto 31

2 Ambientação das Análises de Sistemas Secundários 32

2.1. Tipos de Sistemas Secundários Típicos em uma PWR 32

2.2. Considerações sobre o Espectro de Resposta e a Análise Modal

Espectral 35

2.3. Linha de Pesquisa de Respostas Sísmicas de Sistemas Secundários3¡

3 Representação do Sistema Principal com o Programa Sassi 42

3.1. Análise no domínio da freqüência 42

3.2. O programa SASSI 44

3.3. Sistemas Principais em estudo 50

4 Excitações e Respostas nos Sistemas Secundários 73

4.1. Excitações de projeto $\quad 73$

4.2. Respostas nos sistemas secundários $\quad 79$

5 Roteiro de Cálculo $\quad 98$

5.1. Requisitos iniciais 98

5.2. Roteiro para obtenção de Espectros de Resposta de Projeto de Sistemas Secundários $\quad 99$

5.3. Roteiro para Sistemas Secundários Específicos 106

6 Exemplos de Utilização do Roteiro 107

$\begin{array}{ll}\text { 6.1. Aspectos gerais } & 107\end{array}$ 
6.2. Comparação dos ER obtidos através do roteiro proposto e da forma convencional 108

6.3. Resultados finais nos SS obtidos com a utilização dos ER, ERUP e ERAUP 130

6.4. Comentários sobre os resultados comparados 141

7 Conclusões, comentários e sugestões 142

7.1. Conclusões 142

7.2. Comentários 144

7.3. Sugestões 146

8 Referências 149

ANEXO 1: Características dinâmicas do modelo utilizado para representar : RIS - modelo A1ERE 153

A1.1. Rigidez global da fundação - modelo A1ERE 153

A1.2. Verificações da resposta sísmica do modelo - modelo A1ERE 161

ANEXO 2: Manual do programa SomaMOT 172

A2.1. Introdução 172

A2.2. Dados de Entrada 173

A2.3. Arquivos de entrada e saída 174

A2.4. Lista das rotinas 175

A2.5. Fluxograma simplificado 176

ANEXO 3: Manual de utilização do programa GFiBase 179

A3.1. Introdução 179

A3.2. Dados de Entrada 180

A3.3. Arquivos de entrada e saída 183

A3.4. Lista dos arquivos e das rotinas 184

A3.5. Fluxograma simplificado 185

ANEXO 4: Manual do programa ExConf 187

A4.1. Introdução 187 
A4.2. Descrição do programa

A4.3. Dados de Entrada 188

A4.4. Arquivos de entrada e saída 193

A4.5. Lista dos arquivos e das rotinas 194

A4.6. Fluxograma simplificado 198

ANEXO 5: Manual do programa ExeSASSI 202

A5.1. Introdução 202

A5.2. Descrição do Programa 203

A5.3. Arquivos de entrada e saída 206

A5.4. Lista das rotinas 210

ANEXO 6: Manual para utilização do módulo ACOPLA 211

A6.1. Introdução 211

A6.2. Descrição do programa 212

A6.3. Dados de Entrada $\quad 214$

A6.4. Lista das rotinas 217

A6.5. Fluxograma simplificado 219

$\begin{array}{ll}\text { ANEXO 7: Glossário } & 221\end{array}$ 


\section{Lista de figuras}

Figura 1.1 - Visão esquemática das barreiras de proteção de uma Usina PWR (fonte: $\mathrm{ETN}$ )

Figura 2.1 - Visão esquemática funcionamento de uma Usina PWR (fonte: ETN)

Figura 3.1 Modelo de subestruturação do volume flexível. (a)Sistemas Total (b)Sítio original, com indicação dos nós da fundação (c)Estrutura, com indicação dos nós da estrutura e de interação.

(Fonte:

manual teórico do SASSI2000)

45

Figura 3.2 - Vista esquemática do modelo A3Reator - (fonte: SAMPAIO 1999) 51

Figura 3.3 - Vista esquemática dos elementos da laje de fundo - modelo A3Reator

(fonte: SAMPAIO 1999)

Figura 3.4 - FT para os nós 177/178 - excitação horizontal - modelo A3Reator 53

Figura 3.5 - FT para os nós 177/178 - excitação vertical - modelo A3Reator 53

Figura 3.6 - Vista esquemática do prédio do reator - ERE 55

Figura 3.7 - Esquema em corte das estruturas dos prédio do reator e de segurança 56

Figura 3.8 - Esquema do modelo dos prédios do reator e de segurança 58

Figura 3.9 - Esquema do modelo da fundação 61

Figura 3.10 - FT de acelerações - ERE - SB - excitação em X1 - onda SV 63

Figura 3.11 - FT de acelerações - ERE - SC - excitação em X1 - onda SV 63

Figura 3.12 - FT de acelerações - ERE - RIS topo - excitação em X1 - onda SV

64

Figura 3.13 - FT de acelerações - ERE - RIS +6.95m - excitação em X1 - onda SV 64

Figura 3.14 - FT de acelerações - ERE - base - excitação em X1 - onda SV 65

Figura 3.15 - FT de acelerações - ESG - excitação em X1 - onda SV 65

Figura 3.16 - FT de acelerações - ERE - SB - excitação em X2 - onda SH 66

Figura 3.17 - FT de acelerações - ERE - SC - excitação em X2 - onda SH 67

Figura 3.18 - FT de acelerações - ERE - RIS topo - excitação em X2 - onda SH 
Figura 3.19 - FT de acelerações - ERE - RIS +6.95m - excitação em X2 - onda $\mathrm{SH}$

Figura 3.20 - FT de acelerações - ERE - base - excitação em X2 - onda SH 68

Figura 3.21 - FT de acelerações - ESG - base - excitação em X2 - onda SH 69

Figura 3.22 - FT de acelerações - ERE - SB - excitação em X3 - onda P 70

Figura 3.23 - FT de acelerações - ERE - SC - excitação em X3 - onda P 70

Figura 3.24 - FT de acelerações - ERE - RIS topo - excitação em X3 - onda P 71

Figura 3.25 - FT de acelerações - ERE - RIS +6.95m - excitação em X3 - onda P

Figura 3.26 - FT de acelerações - ERE - base - excitação em X3 - onda P 72

Figura 3.27 - FT de acelerações - ESG - base - excitação em X3 - onda P 72

Figura 4.1 - Espectro de Resposta de Projeto - ERP - amortecimento 7\% 74

Figura 4.2 - Função Densidade de Espectro de Potência de Projeto - FDEPP 75

Figura 4.3 - THD7N7 - excitação na base atuando na direção X 77

Figura 4.4 - THD7N47 - excitação na base atuando na direção Y 77

Figura 4.5 - THD7N17 - excitação na base atuando na direção Z 77

Figura 4.6 - THD7N7 - Amplitude dos Coeficientes de Fourier - direção X 78

Figura 4.7 - THD7N47 - Amplitude dos Coeficientes de Fourier - direção Y 78

Figura 4.8 - THD7N17 - Amplitude dos Coeficientes de Fourier - direção Z 78

Figura 4.9 - A3Reator - Nó 178 (elev. $+29,15 \mathrm{~m}$; R=39m) FT resultante para a direção vertical $Z$

Figura 4.10 - A3Reator - Nó 178 (elev. +29,15m; R=39m) ERZ - soma resultante para a direção vertical $Z \mathrm{ERZ}-\mathrm{mcX}$ - para excitação em X THD7N7 ERZ - mcZ - para excitação em Z - THD47N7 85

Figura 4.11 - Esquema de transferência do movimento de controle no PC para um ponto no interior da estrutura: SASSI e SomaMOT 88

Figura 4.12 - SS representado por um S1GL apoiado no ponto s da estrutura 89 Figura 4.13 - comparação típica entre o ER e ERUP - excitação horizontal em X A3Reator - nó 178 (elev. $+29,15 \mathrm{~m} \mathrm{R}=39 \mathrm{~m})-\xi=3 \% ; \mathrm{p}=84 \%$ 90

Figura 4.14 - A3Reator - ER (nó 178 elev.+29,15m R=39m) ERAUP (SA6 $27,5 \mathrm{t})$ e $(\mathrm{SA} 7-13,8 \mathrm{t}) \quad \xi=3 \% ; \mathrm{p}=84 \%$

Figura 4.15 - A3Reator - ER (nó 176 elev +16,65m) e ERAUP (SA4 - 27,5t) e 
(SA5 -13,8t) $\xi=3 \% ; \mathrm{p}=84 \%$

Figura 4.16 - ER e ERNL - A3Reator, nó 176 elev. $+16,65 m-\xi=3 \%$ para SS específico com Fator de escoamento C=0,4 e amortecimento SS 7\% 97

Figura 5.1 -Roteiro de cálculo e obtenção de ER, ERUP e ERAUP

Figura 6.1 - Vista esquemática de um circuito primário de 2 "loops" de uma usina tipo PWR (fonte: ETN)

109

Figura 6.2 - Vista esquemática do modelo da RIS para a obtenção dos espectros de resposta nos pontos de apoio do RPV, RCP e SG

Figura 6.3 - A1ERE - RPV - elev. +6,95m - nó 116 Comparação entre Espectros de Resposta, ER $-\xi=4 \%$ - obtidos deterministicamente SASSI2000 x STRUDYN

Figura 6.4 - A1ERE - RPV - elev. +6,95m - nó 116 - direção horizontal X Comparação entre os Espectros de Resposta: ER; ERUP; ERAUP - $\xi=4 \%$ $; p=84 \%$

Figura 6.5 - A1ERE - RPV - elev. +6,95m - nó 116 - direção horizontal Y Comparação entre os Espectros de Resposta: ER; ERUP; ERAUP - $\xi=4 \%$ $; p=84 \%$

Figura 6.6- A1ERE - RPV - elev. +6,95m - nó 116 - direção vertical Z Comparação entre os Espectros de Resposta: ER; ERUP; ERAUP $-\xi=4 \%$ $; p=84 \%$

114

Figura 6.7 - Vista esquemática da RCP em um circuito primário de uma usina PWR (fonte: ETN)

116

Figura 6.8 - A1ERE - RCP - elev. +3,70m/+6,95m - nós 127/135 Comparação entre Espectros de Resposta, ER, $\xi=4 \%$ - obtidos deterministicamente SASSI2000 x STRUDYN

Figura 6.9 - A1ERE - RCP - elev. +8,26m - nó 135- direção horizontal X Comparação entre os Espectros de Resposta: ER; ERUP; ERAUP - $\xi=4 \%$ $; p=84 \%$

Figura 6.10 - A1ERE - RCP - elev. +8,26m - nó 135- direção horizontal Y Comparação entre os Espectros de Resposta: ER; ERUP; ERAUP - D=4\%; $\mathrm{p}=84 \%$

Figura 6.11 - A1ERE - RCP - elev. +3,70m - nó 127 - direção vertical Z 
Comparação entre os Espectros de Resposta: ER; ERUP; ERAUP - $\xi=4 \%$ $; p=84 \%$

Figura 6.12 - Vista esquemática da suportação típica do SG de um circuito primário de uma usina do tipo PWR (fonte: ETN)

Figura 6.13 - A1ERE - SG - elev. +3,70m/+9,57m - nós 129/136 Comparação entre Espectros de Resposta, ER, $\xi=4 \%$, obtidos deterministicamente SASSI $2000 \times$ STRUDYN

Figura 6.14 - A1ERE - SG - elev. +3,70m/+15,5m - nós 129/138 Comparação entre Espectros de Resposta, ER, $\xi=4 \%$, obtidos deterministicamente SASSI2000 x STRUDYN

Figura 6.15 - A1ERE - SG - elev. +9,5m/+17,5m - nós 136/138 - direção X Comparação entre os tipos de resposta acoplada: ERUP; ERAUP $-\xi=4 \%$; $\mathrm{p}=84 \%$

Figura 6.16- A1ERE - SG - elev. +9,5m/+17,5m - nós 136/138 - direção Y Comparação entre os tipos de resposta acoplada: ERUP $\xi=4 \% ; p=84 \% 125$ Figura 6.17- A1ERE - SG - elev. +3,7m - nó 129 - direção Z Comparação entre os tipos de resposta acoplada: ERUP; $\xi=4 \% ; p=84 \%$

Figura 6.18 - A1ERE - SG - elev.+9,5/+17,5m apoio nos nós 136/138- direção horizontal X Comparação entre os Espectros de Resposta: ER; ERUP; ERAUP $-\xi=4 \% ; \mathrm{p}=84 \%$

Figura 6.19 - A1ERE - SG - elev.+9,5/+17,5m apoio nos nós 136/138- direção horizontal Y Comparação entre os Espectros de Resposta: ER; ERUP; ERAUP $-\xi=4 \% ; \mathrm{p}=84 \%$

Figura 6.20 - A1ERE - SG - elev.+3,70m - apoio no nó 129- direção horizontal Z Comparação entre os Espectros de Resposta: ER; ERUP; ERAUP - $\xi=4 \%$; $\mathrm{p}=84 \%$

Figura 6.20 - Vista esquemática de um modelo de circuito primário de refrigeração do reator com 2 "loops" de uma usina do tipo PWR

Figura 6.21 - Vista esquemática de um modelo para análise do SS acoplado ao SP (Fonte ETN)

Figura A1.1 - FT de deslocamentos para carga FX=1 - variação na direção X 156 Figura A1.2 - FT de deslocamentos para carga FX=1 - variação na direção Y 156 
Figura A1.3 - FT de deslocamentos para carga FY=1 - variação na direção X 157

Figura A1.4 - FT de deslocamentos para carga FY=1 - variação na direção Y 157

Figura A1.5 - FT de deslocamentos para carga FZ=1 - variação na direção X 158

Figura A1.6 - FT de deslocamentos para carga FZ=1 - variação na direção Y 158

Figura A1.7 - Variação do amortecimento e da rigidez global da fundação com a freqüência - translação

Figura A1.8 - Variação do amortecimento e da rigidez global da fundação com a freqüência - rotação 160

Figura A1.9 - FT de acelerações - base ERE - excitação em X1 - onda SV 163

Figura A1.10 - FT de acelerações - base ESG - excitação em X1 - onda SV 163

Figura A1.11 - FT de acelerações - base ERE - excitação em X2 - onda SH 164

Figura A1.12 - FT de acelerações - base ESG - excitação em X2 - onda SH 164

Figura A1.13 - FT de acelerações - base ERE - excitação em X3 - onda P 165

Figura A1.14 - FT de acelerações - base ESG - excitação em X3 - onda P 165

Figura A1.15 - FT acel. - bases ESG (nó 100) e ERE (nó 103)- excitação em X1 - onda SV

Figura A1.16 - FT acel. - bases ESG (nó 100) e ERE (nó 103)- excitação em X2 - onda SH

Figura A1.17 - FT acel. - bases ESG (nó 100) e ERE (nó 103)- excitação em X3 - onda P

Figura A1.18 - FT acel. - centro ERE e borda da fundação - excitação em X1 onda SV

Figura A1.19 - FT acel. - centro ERE e borda da fundação - excitação em X2 onda $\mathrm{SH}$

Figura A1.20 - FT acel. - centro ERE e borda da fundação - excitação em X3 onda $\mathrm{P}$

Figura A1.21 - FT acel. - pontos alinhados em X1 - excitação em X1 - onda SV

Figura A1.22 - FT acel. - pontos alinhados em X2 - excitação em X1 - onda SV

Figura A1.23 - FT acel. - pontos alinhados em X1 - excitação em X2 - onda SH

Figura A1.24 - FT acel. - pontos alinhados em X2 - excitação em X2 - onda SH 
Figura A1.25 - FT acel. - pontos alinhados em X1 - excitação em X3 - onda P

171

Figura A1.26 - FT acel. - pontos alinhados em X2 - excitação em X3 - onda P 


\section{Lista de tabelas}

Tabela 3.1 - Propriedades dos materiais usados nas análises 57

Tabela 3.2 - Correlação dos nós com as massas concentradas do modelo 59

Tabela 3.3 - Freqüências naturais com a estrutura fixa na base $[\mathrm{Hz}] \quad 60$

Tabela 4.1 - Limites recomendados para escolha de análise acoplada 91

$\begin{array}{ll}\text { Tabela } 6.1 \text { - Coeficientes de rigidez global da fundação } & 108\end{array}$

Tabela 6.2 - Freqüências naturais [Hz] e pesos modais do RPV, desacoplado do SP

Tabela 6.3 - Freqüências naturais de vibração [Hz] e pesos modais das RCP, consideradas desacopladas do SP 116

Tabela 6.4 - Freqüências naturais de vibração [Hz] e pesos modais dos SG, considerados desacoplados do SP 122

Tabela 6.5 - Descrição dos casos de comparação 130

Tabela 6.6 - D47 ER STRUDYN x D49 ER SASSI - comparação de acelerações [g]

Tabela 6.7 - D47 ER STRUDYN x D49 ER SASSI - comparação de deslocamentos $[\mathrm{cm}]$

Tabela 6.8 - D47 ER STRUDYN x D49 ER SASSI - comparação de forças nos suportes dos $\mathrm{SGs}-[\mathrm{kN}]$

Tabela 6.9 - D49 ER SASSI x D50 ERUP SASSI x A51 ERAUP comparação de acelerações

Tabela 6.10 - D49 ER SASSI x D50 ERUP SASSI x A51 ERAUP comparação de deslocamentos

Tabela 6.11 - D49 ER SASSI x D50 ERUP SASSI x A51 ERAUP comparação de forças nos suportes dos $\mathrm{SG}-[\mathrm{kN}] \quad 135$

Tabela 6.12 - ERAUP Tipos 1, 2, 3 e 4 - comparação de acelerações [g] 137

Tabela 6.13 - ERAUP Tipos 1, 2, 3 e 4 - comparação de deslocamentos [cm] 137

Tabela 6.14 - ERAUP Tipos 1, 2, 3 e 4 - comparação de forças nos suportes do $\mathrm{SG}$ Forças Normais - [kN]

Tabela 6.15 - Resultados em aceleração obtidos por diferentes métodos - [g] 139 
Tabela 6.16 - Resultados em deslocamento obtidos por diferentes métodos - [cm]

Tabela A1.1 - Pontos utilizados para comparação das FT de deslocamentos 154

Tabela A1.2 - Coeficientes de rigidez global da fundação 159

Tabela A.5.1 - Nomenclatura dos arquivos *.inp. 207

Tabela A5.2 - Nomenclatura dos arquivos *.out gerados 208

Tabela A5.3 - Nomenclatura dos arquivos *.tap gerados. 209 


\section{Lista de símbolos}

\section{Romanos:}

$a, a, a_{i} \quad$ coeficientes constantes

$A, B \quad$ constantes

C

matriz de rigidez global, complexa

$\widetilde{C} \quad$ matriz de amortecimentos

$d_{\max } \quad$ maior distância entre nós de interação

E módulo de elasticidade

$E(t) \quad$ energia total de um sistema oscilatório

$f_{\max }$

maior freqüência de interesse

$f(t) \quad$ força, no domínio do tempo

$F(\omega) \quad$ força, no domínio da freqüência

$F_{s}(\omega) \quad$ espectro de Fourier de uma função temporal $\ddot{u}_{s}(t)$

$H(\omega) \quad$ função de transferência

$\bar{H}_{X}(\omega) \quad$ função de transferência combinada, para a direção $\mathrm{X}$, das excitações aplicadas às direções $\mathrm{X}, \mathrm{Y}$ e Z

$k_{n, j}\left(\omega_{0 i}\right) \quad$ parcela da rigidez de acoplamento, referente ao nó $n$, para a freqüência $\omega_{0 i}$

$k s p_{n, j}\left(\omega_{0 i}\right) \quad$ rigidez local do sistema principal, referente ao nó $\mathrm{n}$, para a freqüência $\omega_{0 i}$

K matriz de rigidez complexa

$K\left(\omega_{0 i}\right) \quad$ rigidez global de acoplamento, para a freqüência $\omega_{0 i}$

$\widetilde{K} \quad$ matriz de rigidez, complexa

$L_{T}\left(t^{*}\right) \quad$ probabilidade de nenhuma ultrapassagem pela barreira durante o intervalo de tempo $\left[0, t^{\star}\right)$

$m \quad$ massa 


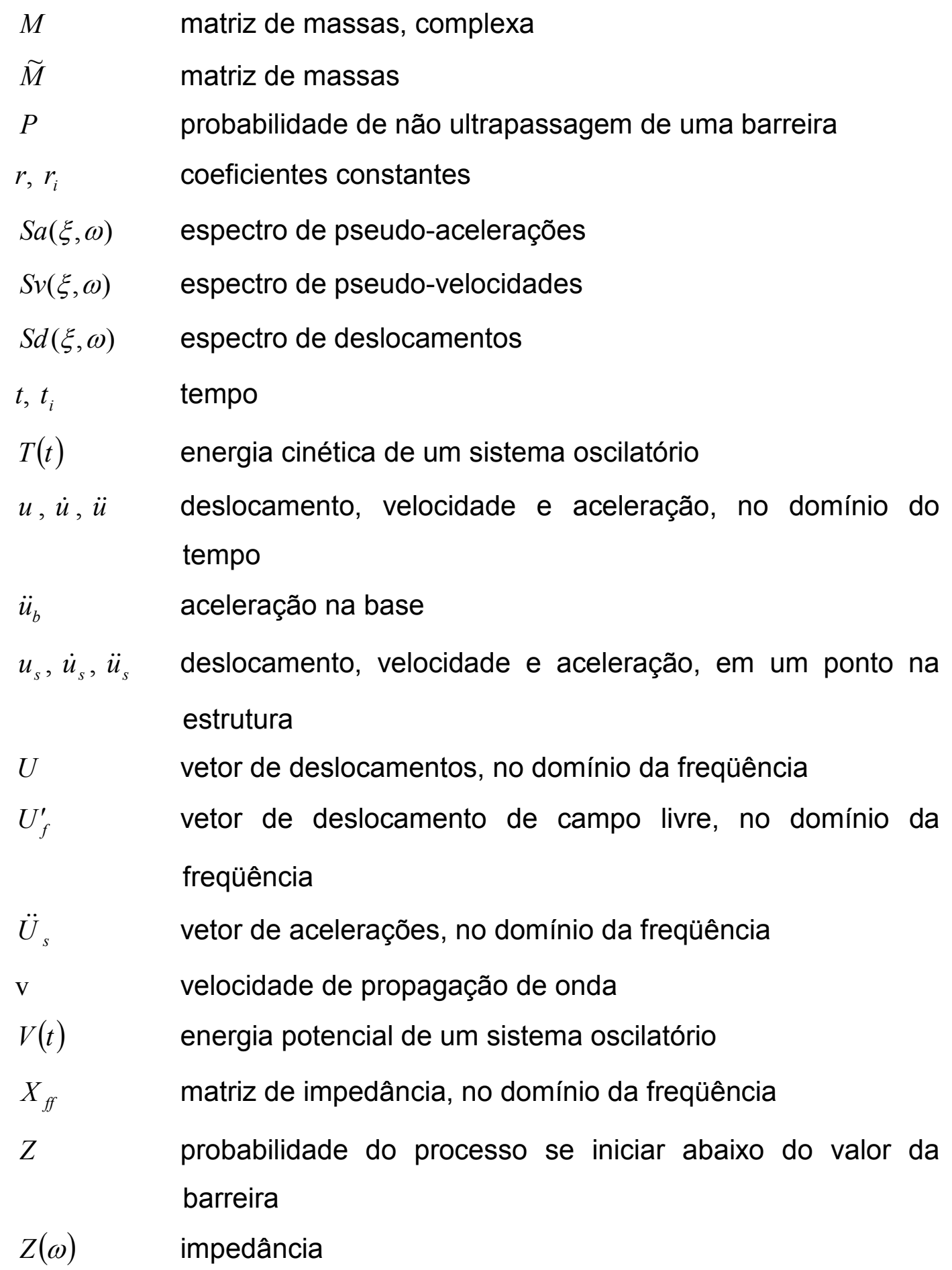




\section{Gregos:}

$\alpha$

$\beta$

$\Delta \omega$

$\xi$

$\lambda_{i}$

$\varsigma$

$\gamma$

$\tau$

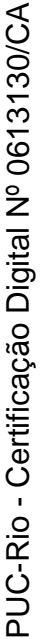

$\omega_{D}$

$\Phi$

razão de decréscimo da probabilidade de primeira ultrapassagem

razão entre freqüências

intervalo de freqüências

amortecimento

momento espectral de i-ésima ordem

deslocamento

peso específico

intervalo de tempo

coeficiente de Poisson

freqüência

freqüência natural

freqüência natural do sistema amortecido

modo de vibração, deslocamentos

$\Phi_{u_{s}}(\omega) \quad$ Função Densidade de Espectro de Potência para a função $\ddot{u}_{s}(t)$

$\eta, \dot{\eta}, \ddot{\eta} \quad$ deslocamento, velocidade e aceleração de um oscilador de 2GL 


\section{Lista de abreviaturas}

A1ERE nome de modelo de edifício do reator utilizado como exemplo

A3Reator nome de modelo de edifício do reator utilizado como exemplo

C Fator de escoamento

CQC Combinação quadrática dos modos

D Amortecimento

ER Espectro de resposta no interior da estrutura

ERAUP Espectro de resposta acoplada uniformemente provável

ERE edifício do reator

ERNL Espectro de resposta não linear

ERP Espectro de Resposta de Projeto

ERUP Espectro de resposta uniformemente provável

ESG edifício de segurança

ETN Eletronuclear

FDEP Função densidade de espectro de potência

FDEPP Função densidade de espectro de potência de projeto

FT Função de transferência

FTD Função de transferência de deslocamentos

GL Grau de liberdade

IMR Rotinas para consideração da influência dos modos rígidos

L onda de superície, do tipo de Lowe

MPRS "Multi Point Response Spectra" - análise modal espectral com excitação múltipla

OBE "Operating Basis Earthquake" - Terremoto de operação de projeto

$\mathrm{p} \quad$ probabilidade de não ultrapassagem

$P \quad$ onda de corpo de compressão, do tipo $P$

PWR "Pressurized Water Reactor" - Reator a água pressurizada

$\mathrm{R} \quad$ onda de superície, do tipo de Rayleigh 
RCL "Reactor Coolant Loop" - Circuito primário de refrigeração do reator

RCP "Reactor Coolant Loop" - Bomba principal do circuito primário de refrigeração do reator

RIS "Reactor Internal Structure" - Estrutura interna do edifício do reator

RPV "Reactor Pressure Vessel" - Vaso de pressão para o núcleo do reator

S1GL Sistema com um grau de liberdade

S2GL Sistema com dois graus de liberdade

SASSI "System for Analysis of Soil-Structure Interaction" - Sistema de programas para análise de interação solo-estrutura

SB

$\mathrm{SC}$

SG "Shield Building" - Estrutura de proteção externa "Steel Containment" - Estrutura de aço de contenção "Steam Generator" - Gerador de Vapor

$\mathrm{SH}$ componente horizontal da onda de corpo de cisalhamento, do tipo $S$

$\mathrm{SP}$

Sistema principal

SS

Sistema secundário

SSE

"Safe Shutdown Earthquake" - Terremoto de desligamento seguro

SSS Sistema secundário simplificado

SV componente vertical da onda de corpo de cisalhamento, do tipo $S$

SVGL Sistema com vários graus de liberdade

TF Transformada de Fourier

UHRS Uniform Hazard Response Spectra" - Espectro de resposta de projeto uniformemente provável

URRS "Uniform Risk Response Spectra" - Espectro de resposta de projeto de risco uniforme

$v_{p} \quad$ velocidade de propagação da onda $P$

$v_{\mathrm{S}} \quad$ velocidade de propagação da onda $S$

$\mathrm{X} 1, \mathrm{X} 2, \mathrm{X} 3$ Direções ortogonais do sistema global de eixo X, Y e Z 


\section{Lista de programas}

SASSI Sistema de programas para análise de interação soloestrutura

SITE Módulo do SASSI para análise do movimento de campo livre

POINT Módulo do SASSI para calcular matriz de impedância

MOTOR Módulo do SASSI para análise de forças harmônicas aplicadas

HOUSE Módulo do SASSI para gerar modelo de elementos finitos

ANALYS Módulo do SASSI para solução da equação do movimento

COMBIN Módulo do SASSI para combinação de FT

MOTION Módulo do SASSI para obtenção das respostas dinâmicas a uma excitação

ExeSASSI Gerenciador para execução dos módulos do SASSI linguagem visual Basic VB6

SomaMOT programa em FORTRAN para somar resultados do MOTION

ACOPLA Módulo do ExeSASSI para possibilitar análises acopladas

GFiBase programa em $\mathrm{C}++$ para gerar FDEP nas posições escolhidas

ExConf programa em C++ para fazer análise probabilística

ACS-SASSI versão comercial do SASSI, pela firma Advanced Computed Softwares

SHAKE programa para análise iterativa de campo-livre

STRUDYN programa para análise análise estrutural dinâmica e estática

DYNRES módulo do STRUDYN para gerar espectros de resposta

ANSYS programa geral para análise de tensões, lineares e não lineares

PosExeSassi modelo de planilha de cálculo, MS-EXCEL, para pósprocessamento do ExeSASSI

PlotaFT macro da planilha PosExeSASSI.xlt, em VB, para plotar FT

PloaEsp macro da planilha PosExeSASSI.xlt, em VB, para plotar espectros e FDEP 